Research

Open Access

\title{
The incidence of sub-optimal sedation in the ICU: a systematic review
}

\author{
Daniel L Jackson ${ }^{1}$, Clare W Proudfoot ${ }^{2}$, Kimberley F Cann² and Tim S Walsh ${ }^{3}$
}

\begin{abstract}
${ }^{1}$ GE Healthcare, Pollards Wood, Nightingales Lane, Chalfont St. Giles, Bucks, HP8 4SP, UK
${ }^{2}$ Heron Evidence Development Ltd, Building 210A, Butterfield Technology and Business Park, Luton, LU2 8DL, UK

${ }^{3}$ Royal Infirmary of Edinburgh, 51 Little France Crescent, Old Dalkeith Road, Edinburgh, EH16 2SA, UK
\end{abstract}

Corresponding author: Daniel L Jackson, Daniel.Jackson@ge.com

Received: 20 Jul 2009 Revisions requested: 29 Sep 2009 Revisions received: 12 Oct 2009 Accepted: 16 Dec 2009 Published: 16 Dec 2009

Critical Care 2009, 13:R204 (doi:10.1186/cc8212)

This article is online at: http://ccforum.com/content/13/6/R204

(C) 2009 Jackson et al.; licensee BioMed Central Ltd.

This is an open access article distributed under the terms of the Creative Commons Attribution License (http://creativecommons.org/licenses/by/2.0), which permits unrestricted use, distribution, and reproduction in any medium, provided the original work is properly cited.

\begin{abstract}
Introduction Patients in intensive care units (ICUs) are generally sedated for prolonged periods. Over-sedation and undersedation both have negative effects on patient safety and resource use. We conducted a systematic review of the literature in order to establish the incidence of sub-optimal sedation (both over- and under-sedation) in ICUs.

Methods We searched Medline, Embase and CINAHL (Cumulative Index to Nursing and Allied Health Literature) online literature databases from 1988 to 15 May 2008 and handsearched conferences. English-language studies set in the ICU, in sedated adult humans on mechanical ventilation, which reported the incidence of sub-optimal sedation, were included. All abstracts were reviewed twice by two independent reviewers, with all conflicts resolved by a third reviewer, to check that they met the review inclusion criteria. Full papers of all
\end{abstract}

included studies were retrieved and were again reviewed twice against inclusion criteria. Data were doubly extracted. Study aims, design, population, comparisons made, and data on the incidence of sub-optimal, optimal, over-sedation or undersedation were extracted.

Results There was considerable variation between included studies in the definition of optimal sedation and in the scale or method used to assess sedation. Across all included studies, a substantial incidence of sub-optimal sedation was reported, with a greater tendency toward over-sedation.

Conclusions Our review suggests that improvements in the consistent definition and measurement of sedation may improve the quality of care of patients within the ICU.

\section{Introduction}

The majority of mechanically ventilated patients within the intensive care unit (ICU) receive sedative drugs. Sedation is administered to ensure patient comfort, reduce anxiety, and facilitate treatments. Optimising sedation management is recognised as important in improving patient outcomes [1]. Under-sedated patients may become agitated and distressed and are at risk of adverse events such as extubation [2-4], whereas over-sedation can prolong time to recovery $[1,5]$.

Assessment of sedation level is carried out mainly by nurses or critical care physicians by assessing patient responses to simple stimuli. Sedation scales such as the Ramsay scale or the Richmond Agitation-Sedation Scale (RASS) are widely used [6-8]. However, there is no universally accepted standard, and this can make comparison between different studies or ICUs difficult [2]. Furthermore, some of these scales have not been fully validated in ICU patients [4]. Recently, devices such as the bispectral index monitor (BIS), which aim to assess sedation levels more objectively, have been introduced. However, most studies of BIS have been performed in surgical settings, and to date its effectiveness is not fully proven [8-10].

Available guidelines on sedation typically provide limited guidance on optimal sedation monitoring and levels. This is at least partly because optimal sedation levels differ between patients according to their clinical circumstances, and therefore sedation practice is ideally individually tailored to each patient, as recommended by several guidelines [2,11,12]. However, among guidelines that do recommend an optimal level of 
sedation, there are discrepancies, indicating a lack of consensus on this issue. For example, of a survey of available guidelines, one [13] recommended a sedation level of 2 or 3 on the Ramsay scale, whereas one recommended a goal of RASS -3 for an intubated patient [14] and a second recommended a goal of RASS 0 to -2 [15]. A number of guidelines stress the importance of establishing a set protocol for the sedation of ICU patients $[16,17]$ but do not set out such a protocol in detail, leaving it to individual institutions, and more recent guidelines recognise the benefit of regular (daily) interruption of sedation for eligible patients $[11,14,18,19]$ within sedation protocols.

It is recognised that optimising sedation practice is a recognised quality marker for intensive care treatment, and procedures designed to optimise patient sedation state, such as daily sedation breaks and more frequent monitoring, are key elements of recent quality improvement initiatives. However, despite these recent efforts to improve the quality of sedation practice in the ICU, the epidemiology of sedation, and specifically the prevalence of over- or under-sedation, is unclear. To investigate this further, we carried out a systematic review of the publicly available literature to identify the reported incidence of sub-optimal sedation.

\section{Materials and methods Searching}

Medline, Embase and the Cumulative Index to Nursing and Allied Health Literature (CINAHL) databases were searched from 1988 to 15 May 2008 using terms for sedation, ICU, sedation quality management, and sub-optimal sedation. The standard Scottish Intercollegiate Guidance Network (SIGN) filters for randomised controlled trials (RCTs), economic studies and observational studies [20] were combined to capture all study designs relevant to the study question. Full details of the search strategy used are available from the authors on request. Conference proceedings from 2005 through 2008 were hand-searched for relevant studies. All results were uploaded into a bespoke internet SOL (structured query language)-based database.

\section{Selection criteria}

Inclusion of studies was according to a predetermined set of criteria. To be included, studies had to be in adult humans who were sedated and undergoing mechanical ventilation within the ICU and furthermore had to report the incidence of suboptimal sedation, over- or under-sedation, or of optimal sedation, as defined by the study. Studies that reported the impact of sedation practice on outcomes were also included; these data are reported separately. In addition, short-term studies (including only patients sedated less than 24 hours) were excluded. Only English-language studies were included. To check that they met the review inclusion criteria, all abstracts were reviewed twice by two independent reviewers, with all conflicts resolved by a third reviewer. Full papers of all included studies were retrieved and were again reviewed twice to ensure that they met inclusion criteria. Studies included at this stage were classified as to which aspect of the review question they met, and appropriate data were extracted, summarised and analysed.

\section{Data extraction}

Data were extracted by two reviewers and checked by a third reviewer against the original studies. For all studies, the following data were extracted: country, sponsor, study design, patient population, objective, number of patients in the study, details of comparisons made (such as between different treatment arms or between different sedation monitoring systems), and the proportion of measurements, patients, or time in which patients were judged to be optimally sedated, sub-optimally sedated, over-sedated, or under-sedated.

\section{Quantitative data synthesis}

Due to the wide range of included study types, no studies were suitable for quantitative data synthesis.

\section{Results}

\section{Systematic review study flow}

The flow of studies through the systematic review is documented in the QUOROM (Quality of Reporting of Meta-Analyses) diagram in Figure 1. Seventy-five primary and seven secondary studies met the inclusion criteria. Of these, $18 \mathrm{did}$ not provide any data; either they did not contain data on the outcomes extracted in this review or they did not provide these data in quantitative form. Thirty-six studies reported data on the incidence of sub-optimal sedation. The remainder reported the impact of sedation practice on outcomes; these data are reported separately. Of the included studies, three were

\section{Figure 1}

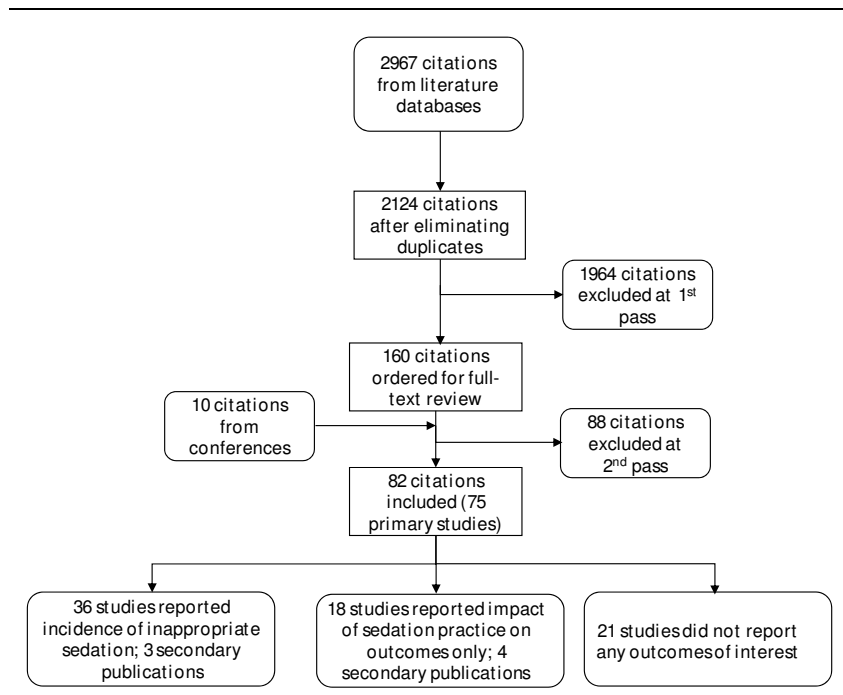

The QUOROM (Quality of Reporting of Meta-Analyses) diagram illustrates the flow of studies through the systematic review. 
cohort studies that specifically investigated the epidemiology of sedation, 23 were studies investigating anaesthetic drugs (of which 19 were RCTs and four were observational studies), six studies compared sedation monitoring devices or scales (of which one was an RCT and the remainder were observational studies), three studies investigated the introduction of sedation guidelines, and one did not fit any of these categories. The majority of studies (20) were published after 2002, indicating the increasing interest in the practice of sedation quality in recent years, in particular following the publication of updated sedation guidelines from the American College of Critical Care Medicine [2,6].

\section{Definitions of adequate sedation}

To assess the incidence of sub-optimal sedation, it is necessary to consider the definition of what constitutes optimal sedation. We used the definition of optimal sedation (and consequently of what constituted sub-optimal sedation) provided by individual studies due to the fact that optimal sedation levels will vary according to study setting (for example, between neurological ICU and medical ICU).

Across all of the studies, 13 different sedation scales were used to assess sedation quality; additionally, nurse assessment of sedation quality simply as over-sedated, undersedated, or adequate was used three times (Table 1). The Ramsay scale was the most commonly used scale, in 14 stud- ies, with a variant used in a further 7 studies. This is illustrated in Figure 2.

In addition to the variation in scales used to assess sedation, there was variation in the recommended range of optimal sedation levels stated. Sedation requirements obviously differ among patients; nevertheless, the variation in recommended ranges in included studies indicates some uncertainty in what constitutes optimal sedation. Of the studies using the Ramsay scale, recommended ranges were 2 to 3 (recommended in two studies [21,22]), 2 to 4 (two studies [23,24]), 2 to 5 (two studies $[25,26]$ ), 3 to 4 (two studies $[27,28]$ ) and 4 to 5 (one study [29]), while three studies did not recommend specific levels but recommended that levels be optimised for each individual patient [30-32]. This variation was reflected in the other scales used; for studies recommending a modified Ramsay scale, recommended ranges were 1 to 4 [33], 3 to 4 [34], 4 [35], and 5 to 6 (the last range being specifically for seriously injured patients [36]) or targets optimised for each patient $[37,38]$. The stated SAS (Riker Sedation-Agitation Scale) target level was 1 to 3 [39], 4 [40,41], or 3 to 4 [42]. Due to the number of studies recommending that optimal sedation state be determined individually for each patient, there was no comparison possible for other scales.

Figure 2

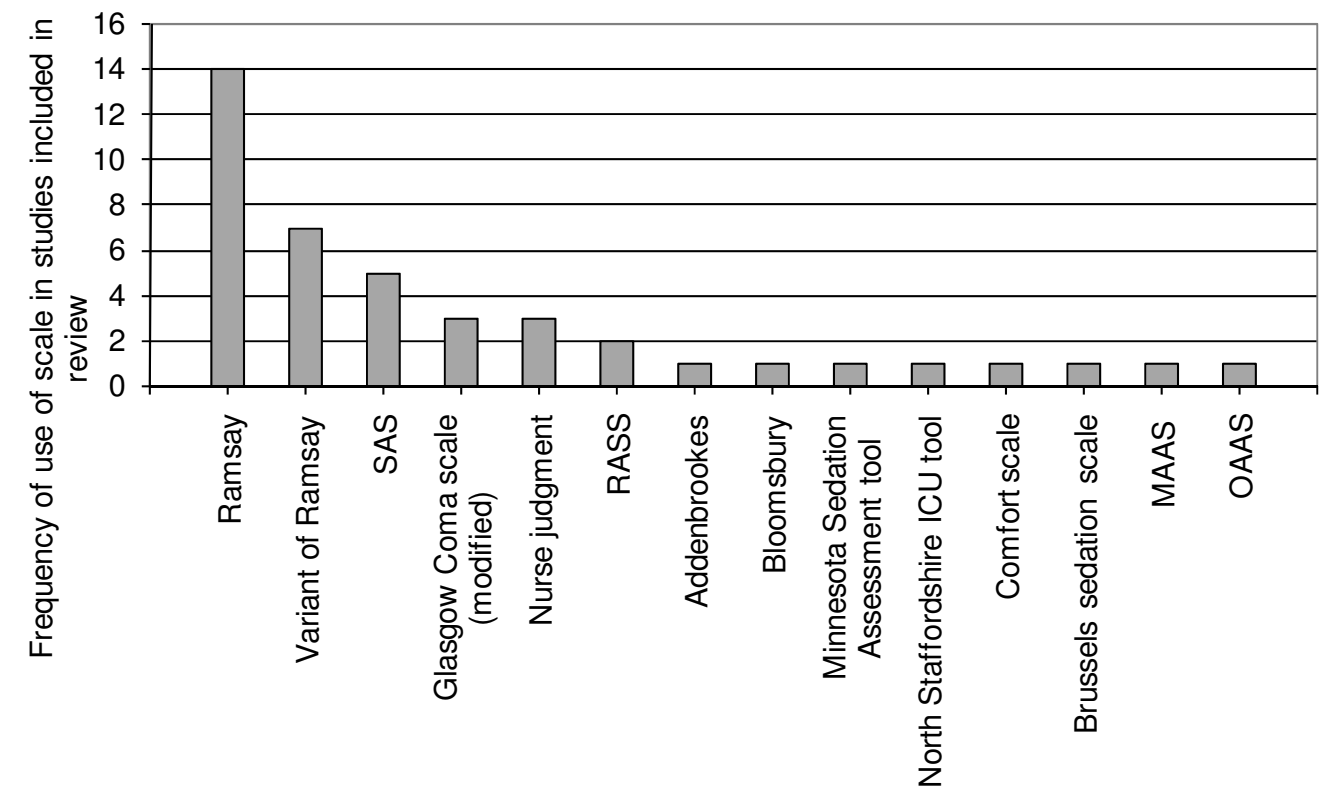

Sedation scale

The frequency with which each sedation scale was used in the studies included in our systematic review. ICU, intensive care unit; MAAS, Motor Activity Assessment Scale; OAAS, Observer's Assessment of Alertness/Sedation Scale; RASS, Richmond Agitation Sedation Scale; SAS, Riker Sedation-Agitation Scale. 
Table 1

Incidence of optimal and sub-optimal sedation in included studies

\begin{tabular}{|c|c|c|c|c|c|c|c|c|c|}
\hline Study & $\begin{array}{l}\text { Study design } \\
\text { and } \\
\text { comparisons } \\
\text { made }\end{array}$ & Number & $\begin{array}{l}\text { Treatment arms } \\
\text { (if relevant) }\end{array}$ & $\begin{array}{l}\text { Incidence of } \\
\text { sub-optimal } \\
\text { sedation }\end{array}$ & $\begin{array}{l}\text { Incidence of } \\
\text { over-sedation }\end{array}$ & $\begin{array}{l}\text { Incidence of } \\
\text { under-sedation }\end{array}$ & $\begin{array}{l}\text { Incidence of } \\
\text { optimal sedation }\end{array}$ & $\begin{array}{l}\text { Sedation scale/ } \\
\text { monitoring } \\
\text { system used }\end{array}$ & $\begin{array}{l}\text { Definition of } \\
\text { optimal sedation }\end{array}$ \\
\hline $\begin{array}{l}\text { Weinert, et al., } \\
2007 \text { [44] }\end{array}$ & Cohort study & 274 & & & $\begin{array}{l}326(2.6 \%) \text { of } \\
12,414 \\
\text { assessments. } \\
111 \text { patients } \\
(40 \%) \text { had } \geq 1 \\
\text { rating of over- } \\
\text { sedation. Patients } \\
\text { were } \\
\text { unarousable/ } \\
\text { minimally } \\
\text { arousable } 32 \% \text { of } \\
\text { the time. }\end{array}$ & $\begin{array}{l}1,731(13.9 \%) \text { of } \\
12,414 \\
\text { assessments. } \\
211(76.2 \%) \text { had } \\
\geq 1 \text { rating of } \\
\text { under-sedation. }\end{array}$ & $\begin{array}{l}10,357(83 \%) \text { of } \\
12,414\end{array}$ & $\begin{array}{l}\text { Minnesota } \\
\text { Sedation } \\
\text { Assessment Tool } \\
-- \text { nurse } \\
\text { assessment }\end{array}$ & $\begin{array}{l}\text { Arousal level 3-5 } \\
\text { (of 6-point scale) }\end{array}$ \\
\hline $\begin{array}{l}\text { Martin, et al., } \\
2006 \text { [30] }\end{array}$ & Cohort study & $\begin{array}{l}305 \text { (from } 220 \\
\text { ICUs) }\end{array}$ & & & $\begin{array}{l}42.6 \% \text { of } 49 \\
\text { patients sedated } \\
24-72 \text { hours, } \\
39.5 \% \text { of } 157 \\
\text { patients sedated } \\
>72 \text { hours, and } \\
43.9 \% \text { of } 57 \\
\text { patients under } \\
\text { weaning had } \\
\text { significantly } \\
\text { deeper sedation } \\
\text { than desired level }\end{array}$ & $\begin{array}{l}5.2 \% \text { of } 157 \\
\text { patients sedated } \\
>72 \text { hours and } \\
3.5 \% \text { of } 57 \\
\text { patients under } \\
\text { weaning had } \\
\text { significantly lower } \\
\text { sedation than } \\
\text { desired level }\end{array}$ & $\begin{array}{l}\text { In patients } \\
\text { sedated }>72 \\
\text { hours, the desired } \\
\text { Ramsay score } \\
\text { was } 0-4 \text { in } 44 \% \text { of } \\
\text { cases -- this was } \\
\text { achieved in } 28 \% \text {; } \\
\text { in } 55 \% \text { of } \\
\text { patients, the } \\
\text { desired value was } \\
4-5 \text {, which was } \\
\text { achieved in } 68 \% \text {; } \\
\text { in } 1 \% \text { of patients, } \\
\text { the desired score } \\
\text { was } 6 \text {, which was } \\
\text { achieved in } 6 \% \text {. }\end{array}$ & Ramsay scale & $\begin{array}{l}\text { Individual to each } \\
\text { patient }\end{array}$ \\
\hline $\begin{array}{l}\text { Payen, et al., } \\
2007 \text { [43] }\end{array}$ & Cohort study & 1,381 & & & $\begin{array}{l}258(57 \%) \text { of } 451 \\
\text { patients on } \\
\text { sedation day } 2 ; \\
169(48 \%) \text { of } 355 \\
\text { patients on day } 4 ; \\
109(41 \%) \text { of } 266 \\
\text { patients on day } 6\end{array}$ & & & $\begin{array}{l}\text { Multiple: most } \\
\text { commonly } \\
\text { Ramsay, RASS, } \\
\text { Sedation- } \\
\text { Agitation scale }\end{array}$ & $\begin{array}{l}\text { Over-sedation } \\
\text { defined as } \\
\text { Ramsay } 5-6, \\
\text { RASS -5 or --4, } \\
\text { Sedation- } \\
\text { Agitation scale 1- } \\
2\end{array}$ \\
\hline \multirow[t]{2}{*}{$\begin{array}{l}\text { Sandiumenge, et } \\
\text { al., } 2000 \text { [36] }\end{array}$} & $\begin{array}{l}\text { RCT/ } \\
\text { observational } \\
\text { study of sedative } \\
\text { drugs }\end{array}$ & 63 & Midazolam & $\begin{array}{l}19(7 \%) \text { of } 266 \\
\text { hours }\end{array}$ & & & $\begin{array}{l}247(93 \%) \text { of } 266 \\
\text { hours }\end{array}$ & $\begin{array}{l}\text { Modified Ramsay } \\
\text { scale }\end{array}$ & $\begin{array}{l}\text { Equivalent of } \\
\text { Ramsay } 5-6 \text { (for } \\
\text { deep sedation) }\end{array}$ \\
\hline & & & $2 \%$ propofol & $\begin{array}{l}14(9 \%) \text { of } 156 \\
\text { hours }\end{array}$ & & & $\begin{array}{l}142(91 \%) \text { of } 156 \\
\text { hours }\end{array}$ & & \\
\hline $\begin{array}{l}\text { Carrasco, et al., } \\
1993[26]\end{array}$ & $\begin{array}{l}\text { RCT (with } \\
\text { economic study) } \\
\text { of sedative drugs }\end{array}$ & 88 & Midazolam & $\begin{array}{l}18 \% \text { of time } \\
\text { (hours) }\end{array}$ & & & $\begin{array}{l}82 \% \text { of time } \\
\text { (hours) }\end{array}$ & $\begin{array}{l}\text { Ramsay scale; } \\
\text { Glasgow coma } \\
\text { scale (modified by } \\
\text { Cook and Palma) }\end{array}$ & $\begin{array}{l}\text { Ramsay scale 2-5, } \\
\text { Glasgow coma } \\
\text { scale 8-13 }\end{array}$ \\
\hline
\end{tabular}




\section{Incidence of optimal and sub-optimal sedation in included studies}

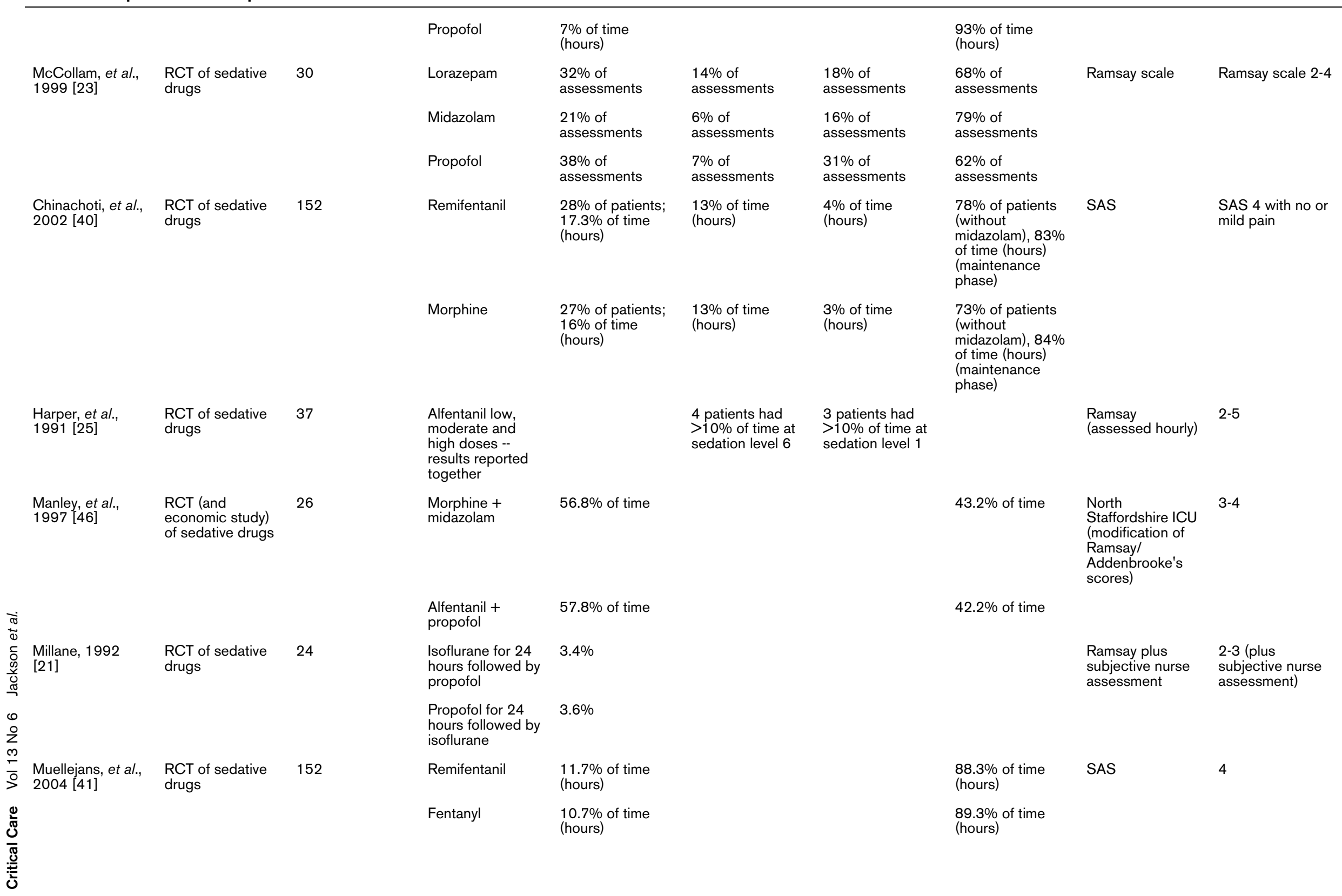




\begin{tabular}{|c|c|c|c|c|c|c|c|c|c|}
\hline \multirow[t]{2}{*}{$\begin{array}{l}\text { Muellejans, et al., } \\
2006 \text { [47] }\end{array}$} & $\begin{array}{l}\text { RCT of sedative } \\
\text { drugs }\end{array}$ & 80 & $\begin{array}{l}\text { Remifentanil -- } \\
\text { propofol }\end{array}$ & $41 \%$ of time & $28 \%$ of time & $13 \%$ of time & $59 \%$ of time & $\begin{array}{l}3 \text { level sedation } \\
\text { score specific to } \\
\text { study }\end{array}$ & Level 2 \\
\hline & & & $\begin{array}{l}\text { Midazolam -- } \\
\text { fentanyl }\end{array}$ & $30 \%$ of time & $19 \%$ of time & $11 \%$ of time & $70 \%$ of time & & \\
\hline \multirow[t]{2}{*}{$\begin{array}{l}\text { Chamorro, et al., } \\
1996 \text { [45] }\end{array}$} & $\begin{array}{l}\text { RCT of sedative } \\
\text { drugs }\end{array}$ & 98 & Propofol & $\begin{array}{l}332 \text { assessments } \\
--3 \% \text { (after first } \\
\text { hour) }\end{array}$ & & & $\begin{array}{l}332 \text { assessments } \\
-76.5 \% \text { effective, } \\
20.5 \% \\
\text { acceptable }\end{array}$ & $\begin{array}{l}\text { Study-specific } \\
\text { (modified } \\
\text { Glasgow coma } \\
\text { scale). Patients } \\
\text { monitored at } 1 \\
\text { and } 6 \text { hours and } \\
\text { then every } 12 \\
\text { hours. }\end{array}$ & $\begin{array}{l}4=\text { effective, } 3= \\
\text { acceptable } \\
\text { less than } 3= \\
\text { ineffective }\end{array}$ \\
\hline & & & Midazolam & $\begin{array}{l}355 \text { assessments } \\
--7.6 \%\end{array}$ & & & $\begin{array}{l}355 \text { assessments } \\
-66.2 \% \text { effective, } \\
26.2 \% \\
\text { acceptable }\end{array}$ & & \\
\hline \multirow[t]{2}{*}{$\begin{array}{l}\text { Barr, et al., } 2001 \\
\text { [34] }\end{array}$} & $\begin{array}{l}\mathrm{RCT} \text { of sedative } \\
\text { drugs }\end{array}$ & 24 & Lorazepam & $51 \%$ of time & $47 \%$ of time & & $49 \%$ of time & Modified Ramsay & $\begin{array}{l}3-4(5-6=\text { over- } \\
\text { sedation) }\end{array}$ \\
\hline & & & Midazolam & $31 \%$ of time & $22 \%$ of time & & $69 \%$ of time & & \\
\hline \multirow[t]{2}{*}{$\begin{array}{l}\text { Finfer, et al., } 1999 \\
\text { [33] }\end{array}$} & $\begin{array}{l}\text { RCT of sedative } \\
\text { drugs }\end{array}$ & 40 & $\begin{array}{l}\text { Diazepam } \\
\text { (intermittent) }\end{array}$ & $\begin{array}{l}9(64.3 \%) \text { of } 14 \\
\text { patients; } 15.0 \% \\
\text { of time (hours) }\end{array}$ & $\begin{array}{l}2.8 \% \text { of time } \\
\text { (hours) }\end{array}$ & $\begin{array}{l}21.1 \% \text { of time } \\
\text { (hours) }\end{array}$ & $\begin{array}{l}5(35.7 \%) \text { of } 14 \\
\text { patients; } \\
85.0 \% \text { of time } \\
\text { (hours) }\end{array}$ & Modified Ramsay & $1-4$ \\
\hline & & & $\begin{array}{l}\text { Midazolam } \\
\text { (continuous) }\end{array}$ & $\begin{array}{l}6(35.3 \%) \text { of } 17 \\
\text { patients; } 40.8 \% \\
\text { of time (hours) }\end{array}$ & $\begin{array}{l}14.8 \% \text { of time } \\
\text { (hours) }\end{array}$ & $\begin{array}{l}0 \% \text { of time } \\
\text { (hours) }\end{array}$ & $\begin{array}{l}11(64.7 \%) \text { of } 17 \\
\text { patients; } \\
59.2 \% \text { of time } \\
\text { (hours) }\end{array}$ & & \\
\hline \multirow[t]{2}{*}{$\begin{array}{l}\text { Richman, et al., } \\
2006 \text { [37] }\end{array}$} & $\begin{array}{l}\text { RCT of sedative } \\
\text { drugs }\end{array}$ & 30 & Midazolam & $\begin{array}{l}\text { Mean } 9.1 \text { hours/ } \\
\text { day (SD 4.9) }\end{array}$ & & & & Modified Ramsay & $\begin{array}{l}\text { Individual to each } \\
\text { patient }\end{array}$ \\
\hline & & & $\begin{array}{l}\text { Midazolam and } \\
\text { fentanyl }\end{array}$ & $\begin{array}{l}\text { Mean } 4.2 \text { hours/ } \\
\text { day (SD } 2.4)\end{array}$ & & & & & \\
\hline \multirow[t]{3}{*}{$\begin{array}{l}\text { Karabinis, et al., } \\
2004 \text { [39] }\end{array}$} & $\begin{array}{l}\text { RCT of sedative } \\
\text { drugs }\end{array}$ & 161 & Remifentanil & $4.4 \%$ of time & & & $\begin{array}{l}95.6 \% \text { of time } \\
\text { (median) }\end{array}$ & SAS & $1-3$ \\
\hline & & & Fentanyl & $1.9 \%$ of time & & & $\begin{array}{l}98.1 \% \text { of time } \\
\text { (median) }\end{array}$ & & \\
\hline & & & Morphine & $1.0 \%$ of time & & & $\begin{array}{l}99.0 \% \text { of time } \\
\text { (median) }\end{array}$ & & \\
\hline $\begin{array}{l}\text { Pandharipande, et } \\
\text { al., 2007 [48], } \\
\text { Pandharipande, et } \\
\text { al., } 2006 \text { [59] }\end{array}$ & $\begin{array}{l}\text { RCT of sedative } \\
\text { drugs }\end{array}$ & 106 & Dexmedetomidine & $\begin{array}{l}20 \% \text { of patients } \\
\text { according to } \\
\text { nurse goals; } 33 \% \\
\text { according to } \\
\text { physician goals }\end{array}$ & $15 \%$ of patients & & $\begin{array}{l}80 \% \text { of patients } \\
\text { within } 1 \text { point of } \\
\text { nurse goal; } 67 \% \\
\text { within } 1 \text { point of } \\
\text { physician goal }\end{array}$ & $\begin{array}{l}\text { RASS, confusion- } \\
\text { assessment } \\
\text { method for the } \\
\text { ICU (CAM-ICU) }\end{array}$ & $\begin{array}{l}\text { Individual to each } \\
\text { patient }\end{array}$ \\
\hline
\end{tabular}




\begin{tabular}{|c|c|c|c|c|c|c|c|c|c|}
\hline & & & Lorazepam & $\begin{array}{l}33 \% \text { of patients } \\
\text { according to } \\
\text { nurse goals; } 45 \% \\
\text { according to } \\
\text { physician goals }\end{array}$ & $33 \%$ of patients & & $\begin{array}{l}67 \% \text { within } 1 \\
\text { point of nurse } \\
\text { goal; } 55 \% \text { within } \\
1 \text { point of } \\
\text { physician goal }\end{array}$ & & \\
\hline \multirow[t]{2}{*}{$\begin{array}{l}\text { Swart, et al., } \\
1999 \text { [50] }\end{array}$} & $\begin{array}{l}\text { RCT of sedative } \\
\text { drugs }\end{array}$ & 64 & Lorazepam & $13 \%$ of time & & & $\begin{array}{l}87.0 \% \text { of time } \\
\text { (SD 10.5) }\end{array}$ & $\begin{array}{l}\text { Addenbrooke's } \\
\text { Hospital's ICU } \\
\text { sedation scale }\end{array}$ & $\begin{array}{l}\text { Individual to each } \\
\text { patient }\end{array}$ \\
\hline & & & Midazolam & $34 \%$ of time & & & $\begin{array}{l}66.2 \% \text { of time } \\
\text { (SD 23.1) }\end{array}$ & & \\
\hline \multirow[t]{2}{*}{$\begin{array}{l}\text { Carson, et al., } \\
2006 \text { [22] }\end{array}$} & $\begin{array}{l}\text { RCT of sedative } \\
\text { drugs }\end{array}$ & 132 & $\begin{array}{l}\text { Intermittent } \\
\text { lorazepam }\end{array}$ & $\begin{array}{l}42.8 \% \text { (ventilator } \\
\text { hours) }\end{array}$ & $\begin{array}{l}37.9 \% \text { (ventilator } \\
\text { hours) }\end{array}$ & $\begin{array}{l}15.1 \% \text { (ventilator } \\
\text { hours) }\end{array}$ & & Ramsay & $2-3$ \\
\hline & & & $\begin{array}{l}\text { Continuous } \\
\text { propofol }\end{array}$ & $\begin{array}{l}49.9 \% \text { (ventilator } \\
\text { hours) }\end{array}$ & $\begin{array}{l}38.6 \% \text { (ventilator } \\
\text { hours) }\end{array}$ & $\begin{array}{l}11.5 \% \text { (ventilator } \\
\text { hours) }\end{array}$ & & & \\
\hline \multirow{2}{*}{$\begin{array}{l}\text { Anis, et al., } 2002 \\
\text { [31], Hall, et al., } \\
2001 \text { [60] }\end{array}$} & $\begin{array}{l}\text { RCT of sedative } \\
\text { drugs }\end{array}$ & 156 & Propofol & $39.8 \%$ of time & $12.0 \%$ of time & $11.2 \%$ of time & $60.2 \%$ of time & Ramsay & $\begin{array}{l}\text { Individual to each } \\
\text { patient }\end{array}$ \\
\hline & & & Midazolam & $56.0 \%$ of time & $18.4 \%$ of time & $8.1 \%$ of time & $44.0 \%$ of time & & \\
\hline \multirow[t]{2}{*}{$\begin{array}{l}\text { Park, et al., } 2007 \\
\text { [49] }\end{array}$} & $\begin{array}{l}\text { RCT of sedative } \\
\text { drugs }\end{array}$ & $\begin{array}{l}134(111 \\
\text { analysed) }\end{array}$ & $\begin{array}{l}\text { Analgesia-based } \\
\text { sedation }\end{array}$ & $50 \%$ of time & & & $\begin{array}{l}50 \% \text { of time on } \\
\text { SIMV (median) }\end{array}$ & $\begin{array}{l}\text { Assessor } \\
\text { judgement }\end{array}$ & $\begin{array}{l}\text { Adequate judged } \\
\text { as awake or easily } \\
\text { rousable }\end{array}$ \\
\hline & & & $\begin{array}{l}\text { Hypnotic-based } \\
\text { sedation }\end{array}$ & $81 \%$ of time & & & $\begin{array}{l}19 \% \text { of time on } \\
\text { SIMV (median) }\end{array}$ & & \\
\hline $\begin{array}{l}\text { Cigada, et al., } \\
2005 \text { [32] }\end{array}$ & $\begin{array}{l}\text { Observational } \\
\text { study of sedative } \\
\text { drugs }\end{array}$ & 42 & $\begin{array}{l}\text { Propofol or } \\
\text { midazolam with } \\
\text { enteral } \\
\text { hydroxyzine with } \\
\text { or without } \\
\text { supplemental } \\
\text { lorazepam. IV } \\
\text { drugs were } \\
\text { tapered after } 48 \\
\text { hours. }\end{array}$ & $\begin{array}{l}36.9 \% \text { of } \\
\text { assessments as } \\
\text { judged by Ramsay } \\
\text { score; } 17 \% \text { by } \\
\text { nurse assessment }\end{array}$ & $\begin{array}{l}421(24.6 \%) \text { of } \\
1,711 \\
\text { assessments } \\
\text { (Ramsay score) } \\
42(7.3 \%) \text { of } 577 \\
\text { assessments } \\
\text { (nurse judgement) }\end{array}$ & $\begin{array}{l}211(12.3 \%) \text { of } \\
1,711 \\
\text { assessments } \\
\text { (Ramsay score) } \\
56(9.8 \%) \text { of } 577 \\
\text { assessments } \\
\text { (nurse judgement) }\end{array}$ & $\begin{array}{l}1,079(63.1 \%) \text { of } \\
1,711 \\
\text { assessments } \\
\text { (Ramsay score) } \\
479(83 \%) \text { of } 577 \\
\text { assessments } \\
\text { (nurse judgement) }\end{array}$ & $\begin{array}{l}\text { Ramsay score } \\
\text { plus nurse } \\
\text { assessment }\end{array}$ & $\begin{array}{l}\text { Adequate } \\
\text { sedation defined } \\
\text { as the } \\
\text { achievement of } \\
\text { the planned } \\
\text { Ramsay score or } \\
\text { nurse judgement } \\
\text { as adequate }\end{array}$ \\
\hline $\begin{array}{l}\text { Barrientos-Vega, } \\
\text { et al., } 2001 \text { [29] }\end{array}$ & $\begin{array}{l}\text { Observational } \\
\text { study of sedative } \\
\text { drugs }\end{array}$ & 51 & $\begin{array}{l}2 \% \text { propofol } \\
\text { (compared with } \\
\text { historical cohort } \\
\text { on } 1 \% \text { propofol -- } \\
\text { not reported here) }\end{array}$ & $\begin{array}{l}8(15.6 \%) \text { of } 51 \\
\text { patients judged } \\
\text { therapeutic failure } \\
\text { on } 2 \% \text { propofol } \\
\text { (inadequate level } \\
\text { of sedation) }\end{array}$ & & & & Ramsay score & $4-5$ \\
\hline $\begin{array}{l}\text { MacLaren, et al., } \\
2007 \text { [42] }\end{array}$ & $\begin{array}{l}\text { Observational } \\
\text { study of sedative } \\
\text { drugs }\end{array}$ & 40 & $\begin{array}{l}\text { Dexmedetomidine } \\
\text { as adjunct to } \\
\text { lorazepam/ } \\
\text { midazolam/ } \\
\text { propofol }\end{array}$ & $\begin{array}{l}35 \% \text { of patients } \\
\text { with } \\
\text { dexmedetomidine; } \\
52 \% \text { without }\end{array}$ & $\begin{array}{l}12(30 \%) \text { patients } \\
\text { with } \\
\text { dexmedetomidine; } \\
9(23 \%) \text { without }\end{array}$ & $\begin{array}{l}4(10 \%) \text { patients } \\
\text { with } \\
\text { dexmedetomidine; } \\
12(30 \%) \text { without }\end{array}$ & $\begin{array}{l}65 \% \text { of patients } \\
\text { with } \\
\text { dexmedetomidine; } \\
48 \% \text { without }\end{array}$ & SAS & $3-4$ \\
\hline
\end{tabular}




\section{Incidence of optimal and sub-optimal sedation in included studies}

\begin{tabular}{|c|c|c|c|c|c|c|c|c|c|}
\hline $\begin{array}{l}\text { Shehabi, et al., } \\
2004 \text { [24] }\end{array}$ & $\begin{array}{l}\text { Observational } \\
\text { study of sedative } \\
\text { drugs }\end{array}$ & 20 & $\begin{array}{l}\text { Dexmedetomidine } \\
\text { with supplemental } \\
\text { midazolam if } \\
\text { required }\end{array}$ & $\begin{array}{l}455(33 \%) \text { of } \\
1,381 \\
\text { assessments }\end{array}$ & $\begin{array}{l}97(7 \%) \text { of } 1,381 \\
\text { assessments } \\
\text { were Ramsay } \\
\text { level } 6\end{array}$ & $\begin{array}{l}137(10 \%) \text { of } \\
1,381 \\
\text { assessments } \\
\text { were Ramsay } \\
\text { level } 1\end{array}$ & $\begin{array}{l}926(67 \%) \text { of } \\
1,381\end{array}$ & Ramsay & $2-4$ \\
\hline \multirow[t]{2}{*}{$\begin{array}{l}\text { Sackey, et al., } \\
2004 \text { [51] }\end{array}$} & $\begin{array}{l}\text { RCT of sedation } \\
\text { devices }\end{array}$ & 40 & $\begin{array}{l}\text { Isoflurane using } \\
\text { AnaConDa }\end{array}$ & $\begin{array}{l}46 \% \text { of time; } \\
\text { nursing staff } \\
\text { estimate } 11 \% \text { of } \\
\text { time }\end{array}$ & $44 \%$ of time & $2 \%$ of time & $\begin{array}{l}54 \% \text { of time; } \\
\text { nursing staff } \\
\text { estimate } 89 \% \text { of } \\
\text { time }\end{array}$ & Bloomsbury scale & -1 to +1 \\
\hline & & & IV midazolam & $\begin{array}{l}41 \% \text {; nursing staff } \\
\text { estimate } 13 \% \text { of } \\
\text { time }\end{array}$ & $37 \%$ of time & $4 \%$ of time & $\begin{array}{l}59 \% \text { of time; } \\
\text { nursing staff } \\
\text { estimate } 87 \% \text { of } \\
\text { time }\end{array}$ & & \\
\hline $\begin{array}{l}\text { Walsh, et al., } \\
2008 \text { [52] }\end{array}$ & $\begin{array}{l}\text { Observational } \\
\text { study of sedation } \\
\text { devices }\end{array}$ & 30 & $\begin{array}{l}\text { All sedated } \\
\text { patients }\end{array}$ & & $\begin{array}{l}137(32.9 \%) \text { of } \\
416 \text { assessments } \\
\text { (Ramsay score 5- } \\
\text { 6) }\end{array}$ & $\begin{array}{l}5(1.2 \%) \text { of } 416 \\
\text { assessments } \\
\text { (Ramsay score } 1)\end{array}$ & & $\begin{array}{l}\text { Entropy Module/ } \\
\text { Modified Ramsay } \\
\text { scale }\end{array}$ & $\begin{array}{l}\text { None stated. } \\
\text { Refers to } \\
\text { guidelines } \\
\text { suggesting } 2-3 \text { is } \\
\text { adequate and } \\
\text { heavy/over- } \\
\text { sedated is } 5-6 \text {. }\end{array}$ \\
\hline $\begin{array}{l}\text { Hernández- } \\
\text { Gancedo, et al., } \\
2006 \text { [28] }\end{array}$ & $\begin{array}{l}\text { Observational } \\
\text { study of sedation } \\
\text { scales }\end{array}$ & 50 & & & $\begin{array}{l}44 \% \text { ( } 66 \text { cases) -- } \\
\text { Ramsay level } 6\end{array}$ & & $25 \%$ (38 cases) & $\begin{array}{l}\text { Ramsay, } \\
\text { Observer's } \\
\text { Assessment of } \\
\text { Alertness and } \\
\text { Sedation }\end{array}$ & Ramsay 3-4 \\
\hline $\begin{array}{l}\text { Roustan, et al., } \\
2005 \text { [27] }\end{array}$ & $\begin{array}{l}\text { Observational } \\
\text { study of sedation } \\
\text { scales }\end{array}$ & 40 & $\begin{array}{l}\text { All sedated } \\
\text { patients -- treated } \\
\text { with midazolam } \\
\text { and morphine }\end{array}$ & & $\begin{array}{l}93(61.6 \%) \text { of } \\
151 \text { records }\end{array}$ & $\begin{array}{l}19(12.6 \%) \text { of } \\
151 \text { records }\end{array}$ & & $\begin{array}{l}\text { Ramsay, Comfort } \\
\text { score, EEG }\end{array}$ & Ramsay 3-4 \\
\hline $\begin{array}{l}\text { McMurray, et al., } \\
2004 \text { [38] }\end{array}$ & $\begin{array}{l}\text { Observational } \\
\text { study of sedation } \\
\text { scales }\end{array}$ & 122 & $\begin{array}{l}\text { Propofol- } \\
\text { containing } \\
\text { regimens }\end{array}$ & $15.6 \%$ of time & $\begin{array}{l}\text { Mean } 5.0 \% \text { of } \\
\text { time (SD 12.7) }\end{array}$ & $\begin{array}{l}\text { Mean } 10.6 \% \text { of } \\
\text { time (SD 14.5) }\end{array}$ & $\begin{array}{l}\text { Mean } 84.4 \% \text { of } \\
\text { time (SD 18.0) }\end{array}$ & Modified Ramsay & $\begin{array}{l}\text { Individual to each } \\
\text { patient }\end{array}$ \\
\hline \multirow[t]{2}{*}{$\begin{array}{l}\text { Detriche, et al., } \\
1999 \text { [53] }\end{array}$} & $\begin{array}{l}\text { Before-after study } \\
\text { of introduction of } \\
\text { sedation protocol }\end{array}$ & 55 & Before & & & $\begin{array}{l}20(30 \%) \text { of } 67 \\
\text { assessment days }\end{array}$ & & $\begin{array}{l}\text { Brussels sedation } \\
\text { scale }\end{array}$ & $3-4$ \\
\hline & & & $\begin{array}{l}\text { After protocol } \\
\text { introduction }\end{array}$ & & & $\begin{array}{l}9(12 \%) \text { of } 77 \\
\text { assessment days }\end{array}$ & & & \\
\hline \multirow[t]{2}{*}{$\begin{array}{l}\text { Costa, et al., } \\
1994 \text { [54] }\end{array}$} & $\begin{array}{l}\text { RCT of controlled } \\
\text { and empirical } \\
\text { sedation }\end{array}$ & 40 & Controlled & $17 \%$ of time & & & $83 \%$ of time & $\begin{array}{l}\text { Ramsay, and } \\
\text { Glasgow coma } \\
\text { scale modified by } \\
\text { Cook and Palma }\end{array}$ & \\
\hline & & & Empirical & $65 \%$ of time & & & $35 \%$ of time & & \\
\hline $\begin{array}{l}\text { MacLaren, et al., } \\
2000 \text { [35] }\end{array}$ & $\begin{array}{l}\text { Before-after } \\
\text { comparison of } \\
\text { sedation protocol }\end{array}$ & 158 & Before & & & $\begin{array}{l}22.4 \% \\
\text { (experience of } \\
\text { anxiety or pain) }\end{array}$ & & Modified Ramsay & 4 \\
\hline
\end{tabular}


Table 1 (Continued)

\section{Incidence of optimal and sub-optimal sedation in included studies}

\begin{tabular}{|c|c|c|c|c|c|c|c|c|}
\hline & & & After & & $\begin{array}{l}11.0 \%(P< \\
0.001)\end{array}$ & & & \\
\hline \multirow[t]{2}{*}{$\begin{array}{l}\text { Tallgren, et al., } \\
2006 \text { [3] }\end{array}$} & $\begin{array}{l}\text { Before-after } \\
\text { comparison of } \\
\text { sedation protocol }\end{array}$ & 53 & $\begin{array}{l}\text { Before } \\
\text { reinforcement }\end{array}$ & & $\begin{array}{l}\text { Median Ramsay } \\
\text { level was } 4 \text { during } \\
\text { the day and } 5 \text { at } \\
\text { night, in contrast } \\
\text { to the study's } \\
\text { stated aim of } \\
\text { Ramsay level } 2-3 \\
\text { during the day } \\
\text { and } 3-4 \text { at night }\end{array}$ & & & Ramsay \\
\hline & & & $\begin{array}{l}\text { After } \\
\text { reinforcement }\end{array}$ & & $\begin{array}{l}\text { Median Ramsay } \\
\text { level was } 4 \text { during } \\
\text { the day and } 5 \text { at } \\
\text { night, in contrast } \\
\text { to the study's } \\
\text { stated aim of } \\
\text { Ramsay level 2-3 } \\
\text { during the day } \\
\text { and 3-4 at night }\end{array}$ & & & \\
\hline $\begin{array}{l}\text { Samuelson, et al., } \\
2007 \text { [61], } \\
\text { Samuelson, et al., } \\
2006 \text { [62] }\end{array}$ & $\begin{array}{l}\text { Observational } \\
\text { study }\end{array}$ & 250 & & $\begin{array}{l}50 \% \text { of patients } \\
\text { had MAAS 0-2 } \\
\text { (although } 2 \text { was } \\
\text { target for study, 0- } \\
1 \text { could be viewed } \\
\text { as over-sedated) }\end{array}$ & $0 \%$ & $\begin{array}{l}39 \% \text { of patients } \\
\text { achieved MAAS } 3 \\
\text { in ventilated } \\
\text { period }\end{array}$ & MAAS & $\begin{array}{l}\text { Stated } 2-3 \text { but } \\
\text { results reported } \\
\text { for patients } \\
\text { achieving } 3\end{array}$ \\
\hline
\end{tabular}

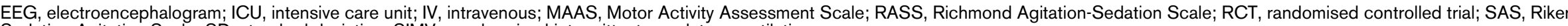
Sedation-Agitation Scale; SD, standard deviation; SIMV, synchronised intermittent mandatory ventilation. 


\section{Incidence of sub-optimal sedation}

Table 1 lists the study design, sedation assessment scale or tool used, and incidence of sub-optimal sedation reported by studies. As stated above, we used individual study definitions of optimal and sub-optimal sedation because of the fact that optimal sedation levels are likely to vary by study setting.

The three observational studies that investigated the epidemiology of sedation were considered to be the most relevant to the study question as their specific aim was to investigate clinical sedation practice rather than practice within the confines of a trial, where more frequent monitoring and the Hawthorne effect could contribute to improving standards.

A survey of practice across 44 ICUs in France also found a high incidence of deep sedation, in $41 \%$ to $57 \%$ of readings over a 6-day period [43]. This study highlighted the risks of prolonged deep sedation, which, however, was not specifically defined as over-sedation. Results from these three studies indicate that $30 \%$ to $60 \%$ of sedation assessments indicate 'deep' or 'over' sedation, although precise description of the prevalence is confounded by imprecise definition or health care worker perceptions. These studies clearly indicate an excess of over-sedation compared with under-sedation.

Martin and colleagues [30] conducted a postal survey of 220 ICUs in Germany. This study found that $42.6 \%$ of patients sedated between 24 and 72 hours and $39.5 \%$ of patients sedated over 72 hours were over-sedated; the incidence of under-sedation was much lower $(<6 \%)$.

In the US-based study of Weinert and colleagues [44], the aim was to compare subjective and objective ratings of sedation. Subjects provided 12,414 sedation assessments and were judged by nurses to be sub-optimally sedated in $17 \%$ of assessments, over-sedated in $2.6 \%$, and under-sedated in $13.9 \%$. Critically, however, patients were unrousable or minimally rousable just under one third of the time, indicating a high incidence of deep sedation. This finding illustrates the importance of the perception of the health care worker or assessor or both in describing the prevalence of sub-optimal sedation.

The remaining included studies comprised studies of sedative drugs [21-26,29,31-34,36,37,39-42,45-50], studies investigating different sedation devices or scales [27,28,38,51,52], and studies looking at the introduction of a sedation guideline or protocol $[3,35,53,54]$. Studies varied by design and aim, by sedatives used, by scales and definitions of sub-optimal sedation used, and by the way incidence was reported (as a proportion of measurements, patients, or time). While these studies did not necessarily have the incidence of sub-optimal sedation as their primary focus, the data in such studies were considered to be of interest to the inclusive scope of this review. Although studies of sedative drugs or of the introduc- tion of guidelines or protocols may not give an accurate estimate of the incidence of sub-optimal sedation within routine clinical practice, they nevertheless show that it does occur and can give an impression of the extent to which it may be a problem, even in settings that could be reasonably expected to be more controlled than in routine practice. The incidence of suboptimal sedation reported in these studies is summarised in Figure 3 (separated by study and treatment arm where relevant). The reported incidence varied from 1\% [39] to $75 \%$ [28], with the majority reporting an incidence of over $20 \%$. The incidence of over- and under-sedation was similarly variable, and figures of between $2.8 \%$ and $44 \%$ for over-sedation [28,33,51] and between $2 \%$ and $31 \%$ for under-sedation $[23,51]$ were reported. A further study [2] that looked at the introduction of a sedation guideline did not record the incidence of sub-optimal sedation but recorded the median Ramsay scale values. These were 4 during the day and 5 at night, in contrast to the study's stated aim of Ramsay levels of 2 to 3 during the day and 3 to 4 at night; this study again noted a possible tendency toward over-sedation of patients. Importantly, there was no change in this tendency before and after reinforcement of the guideline, suggesting that this was insufficient to improve sedation practice [3].

\section{Discussion}

Our systematic review identified few studies that specifically described the epidemiology of sedation during ICU care. Description of the incidence of sub-optimal sedation and overand under-sedation was difficult due to variation in the use of these terms within individual studies. Overall, available data suggest a high incidence of over-sedation in ICUs, potentially present at $40 \%$ to $60 \%$ of assessments. A lower reported incidence of sub-optimal sedation across most studies suggests that health care workers consider deep levels of sedation appropriate for many patients.

The quality of published studies was low. There was wide variation in the method used to assess sedation state, the frequency of measurement, and the stated response to evaluations. In addition, the completeness of data in relation to entire ICU populations was usually not stated, introducing the potential for selection bias. Only three cohort studies were found. The importance of selection or inclusion bias was lowest with this study design. All of these indicated a substantial incidence of sub-optimal sedation, with over-sedation being more common (33\% to $57 \%$ ). Notably, one study reported that nurse assessment of sedation found a low incidence of over-sedation, which appeared at odds with the fact that in one third of measurements patients were unrousable or minimally rousable. A difference in perceptions of what constitutes optimal sedation between different health care worker groups and between individual health care workers is also likely to affect the reported incidence of sub-optimal sedation. This finding emphasises the importance of using sedation-assessment methods that have high validity and low inter-rater varia- 


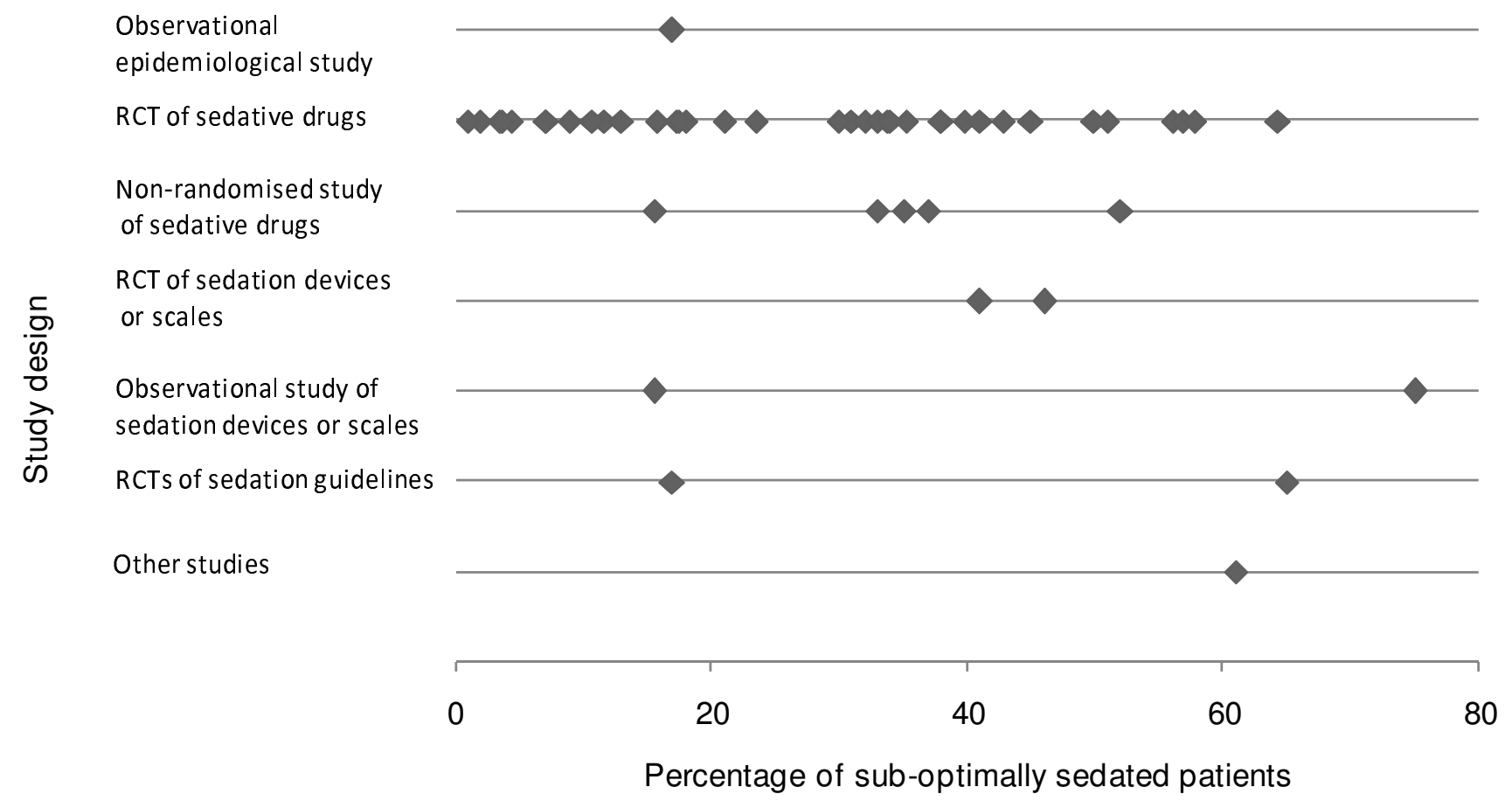

Incidence of sub-optimal sedation across included studies. The plot shows the percentage of measurements, patients, or time in which patients were sub-optimally sedated according to each included study's definition of optimal sedation and measurements reported. Studies are grouped by study design. Where more than one group was reported by a study (for example, a comparison of two different treatment arms), separate points are shown for each group. RCT, randomised controlled trial.

bility. Many of the scales in current use have not been subjected to formal evaluation. The RASS has been shown to have good construct validity and high levels of consistency among health care workers [55] but was used infrequently in the published literature.

When non-cohort studies were considered, a wide range of incidence of sub-optimal sedation levels was reported (from $1 \%$ to $75 \%$ ). These studies were randomised or non-randomised trials of the efficacy of sedative drugs [21-26,29,31$34,36,37,39-42,45-50]$, or were studies that evaluated sedation devices or scales $[27,28,38,51,52]$ to monitor sedation levels, or looked at the introduction of sedation guidelines $[3,35,53,54]$. Overall, the chance of selection and investigator bias, and of study effects, in these studies was high. Drug trials in particular were considered less relevant to our study question as practice within a clinical trial may differ from standard care and is more likely to be controlled. Despite these factors, the majority of studies found an incidence of sub-optimal sedation, using study-specific definitions, of greater than $20 \%$. These studies confirm the findings of the observational cohort studies and suggest high levels of sub-optimal sedation during routine care.
Improving sedation management through sedation protocols and interventions such as daily interruption of sedation is an increasing focus of quality improvement initiatives in critical care in some health care systems $[1,56,57]$. Sedation protocols and scales are increasingly, though not universally, used. A review of German hospitals by Martin and colleagues [6] showed increases in the use of sedation protocols (from $21 \%$ of hospitals to $46 \%$ ) and in the use of sedation scales (from $8 \%$ to $51 \%$ ) over the period of 2002 to 2006 . In Finland, Tallgren and colleagues [3] reported that reinforcing a sedation guideline increased the percentage of expected Ramsay scale recordings made, but only to $71 \%$ of expected recordings that were actually made, indicating that formal sedation assessments were still not carried out as regularly as they should have been. These studies suggest that, despite recent evidence supporting the avoidance of over-sedation [57,58], the use of systematic approaches to measure sedation state and optimise sedation for individual patients is not universal. The high prevalence of sub-optimal sedation and high incidence of over-sedation in published studies indicate potential for significant quality improvement in this aspect of care. This is likely to translate into substantial patient benefit.

There are several limitations to our review. As with any systematic review, studies may have been missed; this review was 
confined to English-language publications and therefore may be biased toward the US and UK in focus. Despite the inclusion of conference searching, there may be relevant grey literature that we did not search. As previously discussed, many of the included studies did not investigate the quality of sedation practice as their primary aim, limiting the relevance of the information provided.

\section{Conclusions}

Our review indicates the poor quality of epidemiological data concerning current sedation practice and the incidence of sub-optimal sedation. A key issue is the standardisation of methods of assessment and definitions of optimal sedation. Despite this, available data suggest that many patients in ICUs are considered sub-optimally sedated and, specifically, that the incidence of over-sedation remains high. The strong associations between sedation practice, especially over-sedation, and adverse patient outcomes suggest that a more uniform approach to monitoring depth and quality of sedation will improve quality of care.

Key messages
The literature shows that, within the intensive care unit
(ICU), a substantial proportion of patients experience
inappropriate levels of sedation (that is, under- or over-
sedation).
- There is a greater tendency toward over-sedation in
particular.
There is a lack of consensus in the literature as to what
constitutes optimal sedation practice within the ICU,
with little standardisation of either assessment methods
or definitions.
Improvements in the definition and measurement of
optimal sedation may have a positive impact on patient
outcomes.

\section{Competing interests}

The systematic review reported in this publication was funded by GE Healthcare (Chalfont St. Giles, UK). DL is an employee of GE Healthcare. CWP and KFC are employed by Heron Evidence Development Ltd (Luton, UK), which was commissioned to undertake research by GE Healthcare. The article processing charge was funded by GE Healthcare. GE Healthcare has developed a device for monitoring consciousness levels in sedated patients TW is collaborating with GE Healthcare in developing a sedation monitoring device. His institution has received research funding from GE for collaborative research, but TW has not gained personally and has not received any direct payment from GE Healthcare. TW holds no shares in GE Healthcare and is not an employee of the company.

\section{Authors' contributions}

DLJ conceived the study and helped with manuscript revisions. CWP designed and performed searches, extracted data and wrote the manuscript draft. KFC researched and wrote the treatment guidelines section and assisted with data extraction for the main systematic review. TW provided expert clinical input and worked on manuscript revisions. All authors read and approved the final manuscript.

\section{Authors' information}

TW is a professor of anaesthetics and critical care at Edinburgh University. DLJ is head of health economics, EMEA (Europe, the Middle East and Africa), at GE Healthcare. CWP is a consultant at Heron Evidence Development Ltd, a health outcomes research consultancy. KFC is a health outcomes analyst at Heron Evidence Development Ltd.

\section{Acknowledgements}

This review was funded by GE Healthcare. The work was performed at Heron Evidence Development Ltd, UK.

\section{References}

1. Fraser GL, Riker RR: Comfort without coma: changing sedation practices. Crit Care Med 2007, 35:635-637.

2. Jacobi J, Fraser GL, Coursin DB, Riker RR, Fontaine D, Wittbrodt ET, Chalfin DB, Masica MF, Bjerke HS, Coplin WM, Crippen DW, Fuchs BD, Kelleher RM, Marik PE, Nasraway SA Jr, Murray MJ, Peruzzi WT, Lumb PD, Task Force of the American College of Critical Care Medicine (ACCM) of the Society of Critical Care Medicine (SCCM), American Society of Health-System Pharmacists (ASHP), American College of Chest Physicians: Clinical practice guidelines for the sustained use of sedative and analgesics in the critically ill adult. Crit Care Med 2002, 30:119-141.

3. Tallgren M, Pettila V, Hynninen M, Tallgren M, Pettila V, Hynninen M: Quality assessment of sedation in intensive care. Acta Anaesthesiol Scand 2006, 50:942-946.

4. Carrasco G: Instruments for monitoring intensive care unit sedation. Crit Care 2000, 4:217-225.

5. Kollef MH, Levy NT, Ahrens TS, Schaiff R, Prentice D, Sherman G: The use of continuous i.v. sedation is associated with prolongation of mechanical ventilation. Chest 1998, 114:541-548.

6. Martin J, Franck M, Sigel S, Weiss M, Spies C: Changes in sedation management in German intensive care units between 2002 and 2006: a national follow-up survey. Crit Care 2007, 11:R124.

7. De Jonghe B, Cook D, Appere-De-Vecchi C, Guyatt G, Meade M, Outin $\mathrm{H}$ : Using and understanding sedation scoring systems: a systematic review. Intensive Care Med 2000, 26:275-285.

8. Pun BT, Dunn J: The sedation of critically ill adults: Part 1: Assessment. The first in a two-part series focuses on assessing sedated patients in the ICU. Am J Nurs 2007, 107:40-48.

9. Vivien B, Di MS, Ouattara A, Langeron O, Coriat P, Riou B: Overestimation of Bispectral Index in sedated intensive care unit patients revealed by administration of muscle relaxant. Anesthesiology 2003, 99:9-17.

10. Avidan MS, Zhang L, Burnside BA, Finkel KJ, Searleman AC, Selvidge JA, Saager L, Turner MS, Rao S, Bottros M, Hantler C, Jacobsohn E, Evers AS: Anesthesia awareness and the bispectral index. N Engl J Med 2008, 358:1097-1108.

11. University of Virginia Health System: Adult critical care sedation guideline. 2005 [http://www.healthsystem.virginia.edu/internet/ e-learning/sedguide.pdf]. Charlottesville, VA: Rector and Board of Visitors of the University of Virginia

12. Mattia C, Savoia G, Paoletti F, Piazza O, Albanese D, Amantea B, Ambrosio F, Belfiore B, Berti M, Bertini L, Bruno F, Carassiti M, Celleno D, Coluzzi F, Consales G, Costantini A, Cuppini F, De Gaudio RA, Farnia A, Finco G, Gravino E, Guberti A, Laurenzi L, Mangione S, Marano M, Mariconda G, Martorano PP, Mediati R, Mercieri M, Mondello E, et al.: SIAARTI recommendations for 
analgo-sedation in intensive care unit. Minerva Anestesiol 2006, 72:769-805.

13. Knape JT, Adriaensen $\mathrm{H}$, van Aken $\mathrm{H}$, Blunnie WP, Carlsson $\mathrm{C}$, Dupont M, Pasch T, Board of Anaesthesiology of the European Union of Medical Specialists: Guidelines for sedation and/or analgesia by non-anaesthesiology doctors. Eur $J$ Anaesthesiol 2007, 24:563-567.

14. University of Pennsylvania Medical Center: Clinical practice guideline manual: analgesia and sedation in the ICU. 2003 [http://www.uphs.upenn.edu/surgery/trauma/scc/protocol/Anal gesia.pdf].

15. Shapiro BA, Warren J, Egol AB, Greenbaum DM, Jacobi J, Nasraway SA, Schein RM, Spevetz A, Stone JR: Practice parameters for intravenous analgesia and sedation for adult patients in the intensive care unit: an executive summary. Society of Critical Care Medicine. Crit Care Med 1995, 23:1596-1600.

16. American College of Critical Care Medicine of the Society of Critical Care Medicine, American Society of Health-System Pharmacists, American College of Chest Physicians: Clinical practice guidelines for the sustained use of sedatives and analgesics in the critically ill adult. Am J Health Syst Pharm 2002, 59:150-178.

17. Feeley K, Gardner A: Sedation and analgesia management for mechanically ventilated adults: literature review, case study and recommendations for practice. Aust Crit Care 2006, 19:73-77.

18. Shapiro MB, West MA, Nathens AB, Harbrecht BG, Moore FA, Bankey PE, Freeman B, Johnson JL, McKinley BA, Minei JP, Moore EE, Maier RV, Inflammation and the Host Response to Injury Large Scale Collaborative Research Project: V. Guidelines for sedation and analgesia during mechanical ventilation general overview. J Trauma 2007, 63:945-950.

19. Dellinger RP, Levy MM, Carlet JM, Bion J, Parker MM, Jaeschke R, Reinhart K, Angus DC, Brun-Buisson C, Beale R, Calandra T, Dhainaut JF, Gerlach H, Harvey M, Marini JJ, Marshall J, Ranieri M, Ramsay G, Sevransky J, Thompson BT, Townsend S, Vender JS, Zimmerman JL, Vincent JL, International Surviving Sepsis Campaign Guidelines Committee; American Association of CriticalCare Nurses; American College of Chest Physicians; American College of Emergency Physicians; Canadian Critical Care Society; European Society of Clinical Microbiology and Infectious Diseases, et al:: Surviving Sepsis Campaign: international guidelines for management of severe sepsis and septic shock: 2008. Crit Care Med 2008, 36:296-327.

20. Scottish Intercollegiate Guidance Network: Methodology search filters. [http://www.sign.ac.uk/methodology/filters.html]. Accessed 2008

21. Millane TA, Bennett ED, Grounds RM: Isoflurane and propofol for long-term sedation in the intensive care unit: a crossover study. Anaesthesia 1992, 47:768-774.

22. Carson SS, Kress JP, Rodgers JE, Vinayak A, Campbell-Bright $S$, Levitt J, Bourdet S, Ivanova A, Henderson AG, Pohlman A, Chang L, Rich PB, Hall J: A randomized trial of intermittent lorazepam versus propofol with daily interruption in mechanically ventilated patients. [See comment]. Crit Care Med 2006, 34:1326-1332

23. McCollam JS, O'Neil MG, Norcross ED, Byrne TK, Reeves ST: Continuous infusions of lorazepam, midazolam, and propofol for sedation of the critically ill surgery trauma patient: a prospective, randomized comparison. [See comment]. Crit Care Med 1999, 27:2454-2458.

24. Shehabi $Y$, Ruettimann $U$, Adamson $H$, Innes $R$, Ickeringill $M$ : Dexmedetomidine infusion for more than 24 hours in critically ill patients: sedative and cardiovascular effects. Intensive Care Med 2004, 30:2188-2196.

25. Harper SJ, Buckley PM, Carr K, Harper SJ, Buckley PM, Carr K: Propofol and alfentanil infusions for sedation in intensive therapy. Eur J Anaesthesiol 1991, 8:157-165.

26. Carrasco G, Molina R, Costa J, Soler JM, Cabre L: Propofol vs midazolam in short-, medium-, and long-term sedation of critically ill patients. A cost-benefit analysis. Chest 1993, 103:557-564.

27. Roustan JP, Valette S, Aubas P, Rondouin G, Capdevila X: Can electroencephalographic analysis be used to determine sedation level in critically ill patients? Anesth Analg 2005, 101:1141-1151. table of contents
28. Hernández-Gancedo $C$, Pestaña D, Peña N, Royo C, Pérez-Chrzanowska $H$, Criado A: Monitoring sedation in critically ill patients: bispectral index, Ramsay and observer scales. Eur J Anaesthesio/ 2006, 23:649-653.

29. Barrientos-Vega R, Sánchez-Soria MM, Morales-Garcia C, CuenaBoy R, Castellano-Hernández M: Pharmacoeconomic assessment of propofol $2 \%$ used for prolonged sedation. Crit Care Med 2001, 29:317-322.

30. Martin J, Franck M, Fischer M, Spies C: Sedation and analgesia in German intensive care units: how is it done in reality? Results of a patient-based survey of analgesia and sedation. Intensive Care Med 2006, 32:1137-1142.

31. Anis $\mathrm{AH}$, Wang $\mathrm{XH}$, Leon $\mathrm{H}$, Hall R, Propofol Study Group: Economic evaluation of propofol for sedation of patients admitted to intensive care units. Anesthesiology 2002, 96:196-201.

32. Cigada M, Pezzi A, Di Mauro P, Marzorati S, Noto A, Valdambrini F, Zaniboni M, Astori M, lapichino G: Sedation in the critically ill ventilated patient: possible role of enteral drugs. Intensive Care Med 2005, 31:482-486.

33. Finfer SR, O'Connor AM, Fisher MM: A prospective randomised pilot study of sedation regimens in a general ICU population: a reality-based medicine study. Crit Care 1999, 3:79-83.

34. Barr J, Zomorodi K, Bertaccini EJ, Shafer SL, Geller E: A doubleblind, randomized comparison of i.v. lorazepam versus midazolam for sedation of ICU patients via a pharmacologic model. [See comment]. Anesthesiology 2001, 95:286-298.

35. MacLaren R, Plamondon JM, Ramsay KB, Rocker GM, Patrick WD, Hall Rl: A prospective evaluation of empiric versus protocolbased sedation and analgesia. Pharmacotherapy 2000, 20:662-672.

36. Sandiumenge Camps A, Sanchez-lzquierdo Riera JA, Toral Vazquez D, Sa Borges M, Peinado Rodriquez J, Alted Lopez E: Midazolam and $2 \%$ propofol in long-term sedation of traumatized critically ill patients: efficacy and safety comparison. Crit Care Med 2000, 28:3612-3619.

37. Richman PS, Baram D, Varela M, Glass PS: Sedation during mechanical ventilation: a trial of benzodiazepine and opiate in combination. Crit Care Med 2006, 34:1395-1401.

38. McMurray TJ, Johnston JR, Milligan KR, Grant IS, Mackenzie SJ, Servin F, Janvier G, Glen JB: Propofol sedation using Diprifusor target-controlled infusion in adult intensive care unit patients. Anaesthesia 2004, 59:636-641.

39. Karabinis A, Mandragos K, Stergiopoulos S, Komnos A, Soukup J, Speelberg B, Kirkham AJ: Safety and efficacy of analgesiabased sedation with remifentanil versus standard hypnoticbased regimens in intensive care unit patients with brain injuries: a randomised, controlled trial. Crit Care 2004, 8:R268-R280

40. Chinachoti T, Kessler P, Kirkham A, Werawatganon T: Remifentanil vs morphine for patients in intensive care unit who need short-term mechanical ventilation. J Med Assoc Thai 2002, 85(Suppl 3):S848-S857.

41. Muellejans B, Lopez A, Cross MH, Bonome C, Morrison L, Kirkham AJ: Remifentanil versus fentanyl for analgesia based sedation to provide patient comfort in the intensive care unit: a randomized, double-blind controlled trial [ISRCTN43755713]. [See comment]. Crit Care 2004, 8:R1-R11.

42. MacLaren R, Forrest LK, Kiser TH: Adjunctive dexmedetomidine therapy in the intensive care unit: a retrospective assessment of impact on sedative and analgesic requirements, levels of sedation and analgesia, and ventilatory and hemodynamic parameters. Pharmacotherapy 2007, 27:351-359.

43. Payen JF, Chanques G, Mantz J, Hercule C, Auriant I, Leguillou JL, Binhas M, Genty C, Rolland C, Bosson JL: Current practices in sedation and analgesia for mechanically ventilated critically ill patients: a prospective multicenter patient-based study. Anesthesiology 2007, 106:687-695. quiz 891-892

44. Weinert CR, Calvin AD, Weinert CR, Calvin AD: Epidemiology of sedation and sedation adequacy for mechanically ventilated patients in a medical and surgical intensive care unit. [See comment]. Crit Care Med 2007, 35:393-401.

45. Chamorro C, de Latorre FJ, Montero A, Sánchez-Izquierdo JA, Jareño A, Moreno JA, Gonzalez E, Barrios M, Carpintero JL, MartínSantos F, Otero B, Ginestal R: Comparative study of propofol versus midazolam in the sedation of critically ill patients: results of a prospective, randomized, multicenter trial. [See comment]. Crit Care Med 1996, 24:932-939. 
46. Manley NM, Fitzpatrick RW, Long T, Jones PW: A cost analysis of alfentanil+propofol vs morphine+midazolam for the sedation of critically ill patients. Pharmacoeconomics 1997, 12:247-255.

47. Muellejans B, Matthey T, Scholpp J, Schill M: Sedation in the intensive care unit with remifentanil/propofol versus midazolam/fentanyl: a randomised, open-label, pharmacoeconomic trial. Crit Care 2006, 10:R91.

48. Pandharipande PP, Pun BT, Herr DL, Maze M, Girard TD, Miller RR, Shintani AK, Thompson JL, Jackson JC, Deppen SA, Stiles RA, Dittus RS, Bernard GR, Ely EW: Effect of sedation with dexmedetomidine vs lorazepam on acute brain dysfunction in mechanically ventilated patients: the MENDS randomized controlled trial. [See comment]. JAMA 2007, 298:2644-2653.

49. Park G, Lane M, Rogers S, Bassett P: A comparison of hypnotic and analgesic based sedation in a general intensive care unit. $\mathrm{Br} J$ Anaesth 2007, 98:76-82.

50. Swart EL, van Schijndel RJ, van Loenen AC, Thijs LG: Continuous infusion of lorazepam versus medazolam in patients in the intensive care unit: sedation with lorazepam is easier to manage and is more cost-effective. Crit Care Med 1999, 27:1461-1465.

51. Sackey PV, Martling CR, Granath F, Radell PJ: Prolonged isoflurane sedation of intensive care unit patients with the Anesthetic Conserving Device. Crit Care Med 2004, 32:2241-2246.

52. Walsh TS, Ramsay P, Lapinlampi TP, Sarkela MO, Viertio-Oja HE, Merilainen PT: An assessment of the validity of spectral entropy as a measure of sedation state in mechanically ventilated critically ill patients. Intensive Care Med 2008, 34:308-315.

53. Detriche O, Berré J, Massaut J, Vincent JL: The Brussels sedation scale: use of a simple clinical sedation scale can avoid excessive sedation in patients undergoing mechanical ventilation in the intensive care unit. [See comment]. Br J Anaesth 1999, 83:698-701.

54. Costa J, Cabre L, Molina R, Carrasco G: Cost of ICU sedation: comparison of empirical and controlled sedation methods. Clin Intensive Care 1994, 5:17-21.

55. Ely EW, Truman B, Shintani A, Thomason JW, Wheeler AP, Gordon S, Francis J, Speroff T, Gautam S, Margolin R, Sessler CN, Dittus RS, Bernard GR: Monitoring sedation status over time in ICU patients: reliability and validity of the Richmond AgitationSedation Scale (RASS). JAMA 2003, 289:2983-2991.

56. Fraser GL, Riker RR: Sedation and analgesia in the critically ill adult. Curr Opin Anaesthesiol 2007, 20:119-123.

57. Kress JP, Pohlman AS, O'Connor MF, Hall JB: Daily interruption of sedative infusions in critically ill patients undergoing mechanical ventilation. [See comment]. N Engl J Med 2000, 342:1471-1477.

58. Girard TD, Kress JP, Fuchs BD, Thomason JW, Schweickert WD, Pun BT, Taichman DB, Dunn JG, Pohlman AS, Kinniry PA, Jackson JC, Canonico AE, Light RW, Shintani AK, Thompson JL, Gordon SM, Hall JB, Dittus RS, Bernard GR, Ely EW: Efficacy and safety of a paired sedation and ventilator weaning protocol for mechanically ventilated patients in intensive care (Awakening and Breathing Controlled trial): a randomised controlled trial. [See comment]. Lancet 2008, 371:126-134.

59. Pandharipande PP, Frizell J, Bun BT, Maze M, Ely EW: Randomized Controlled trial comparing dexmedetomidine with lorazepam to reduce delirium in the ICU. Anesthesiology 2006, 105:A210.

60. Hall RI, Sandham D, Cardinal P, Tweeddale M, Moher D, Wang X, Anis AH: Propofol vs midazolam for ICU sedation: a Canadian multicenter randomized trial. Chest 2001, 119:1151-1159.

61. Samuelson K, Lundberg D, Fridlund B: Stressful memories and psychological distress in adult mechanically ventilated intensive care patients - a 2-month follow-up study. Acta Anaesthesiol Scand 2007, 51:671-678.

62. Samuelson K, Lundberg D, Fridlund B: Memory in relation to depth of sedation in adult mechanically ventilated intensive care patients. Intensive Care Medicine 2006, 32:660-667. 